\title{
An Asymptotic Relation for Conformal Radii of Two Nonoverlapping Domains
}

\begin{abstract}
A. V. Zherdev
Andrey V. Zherdev, https://orcid.org/0000-0003-2282-4169, Saratov State University, 83, Astrakhanskaya Str., Saratov, 410012, Russia; Petrozavodsk State University, 33, Lenin Str., Petrozavodsk, Republic of Karelia, 185910, Russia, jerdevandrey@gmail.com

We consider a family of continuously varying closed Jordan curves given by a polar equation, such that the interiors of the curves form an increasing or decreasing chain of domains. Such chains can be described by the Löwner - Kufarev differential equation. We deduce an integral representation of a driving function in the equation. Using this representation we obtain an asymptotic formula, which establishes a connection between conformal radii of bounded and unbounded components of the complement of the Jordan curve when the bounded component is close to the unit disk.
\end{abstract}

Key words: Löwner-Kufarev equation, conformal radius, asymptotic expansion, nonoverlapping domains.

DOI: https://doi.org/10.18500/1816-9791-2018-18-3-274-283

\section{INTRODUCTION}

We denote by $\mathbb{D}_{r}=\{z \in \mathbb{C}:|z|<r\}$ the open disk of the radius $r>0$ and centered at the origin, $\mathbb{D}=\mathbb{D}_{1}$. Let $\Omega$ be a simply connected domain which is a proper subset of the complex plane and $w_{0} \in \Omega$. According to the Riemann mapping theorem there are a unique number $r>0$ and a unique function $g$ conformally mapping $\Omega$ onto the disk $\mathbb{D}_{r}$ and such that $g\left(w_{0}\right)=0, g^{\prime}\left(w_{0}\right)=1$. This $r$ is called a conformal radius of the domain $\Omega$ with respect to the point $w_{0}$. Let now $\Omega$ be a domain in the extended complex plane with at least two boundary points and $w_{0}=\infty \in \Omega$. There are a unique $r>0$ and a unique function $g$, which is analytic in $\Omega$ except $\infty$, where it has the expansion $g(w)=w+c_{0}+c_{1} w^{-1}+\ldots$, and maps $\Omega$ one-to-one onto $\left\{|z|>\frac{1}{r}\right\}$. This $r$ is called a conformal radius of the domain $\Omega$ with respect to $\infty$. In both cases we denote the conformal radius of $\Omega$ with respect to the point $w_{0}$ by $r\left(\Omega, w_{0}\right)$. Note that if $f$ maps conformally the unit disk $\mathbb{D}$ onto $\Omega \subset \mathbb{C}$ and $f(0)=w_{0}, f^{\prime}(0)>0$, then $r\left(\Omega, w_{0}\right)=f^{\prime}(0)$.

Let $\Omega_{1}, \Omega_{2}$ be disjoint simply connected domains in the extended complex plane, $0 \in \Omega_{1}, \infty \in \Omega_{2}$. Then $r\left(\Omega_{1}, 0\right) r\left(\Omega_{2}, \infty\right) \leqslant 1$, where the equality sign holds if and only if $\Omega_{1}$ and $\Omega_{2}$ are the bounded and unbounded components of the complement of a circle with the center at the origin. This results in a corollary of the theorem about nonoverlapping domains obtained by N. A. Lebedev using the area principle [1]. We will consider the case when $\Omega_{1}$ and $\Omega_{2}$ are the bounded and unbounded components of a closed Jordan curve, respectively. Let $f: \mathbb{D} \rightarrow \Omega_{1}$ and $F:\{|z|>1\} \rightarrow \Omega_{2}$ be conformal maps. The composition $F^{-1} \circ f$ determines a homeomorphism of the unit circle which is called a conformal welding. We refer the reader to the works [2-7].

In the article, we use the Löwner - Kufarev parametric method to establish an asymptotic relation for conformal radii of two nonoverlapping domains. The Löwner equation 
is a differential equation describing a continuously increasing sequence of simply connected domains of a special type, i.e. the so called slit domains [8]. Kufarev [9] and Pommerenke [10] generalized the Löwner equation to a wider class of domains.

Given a chain of simply connected domains $\Omega(t), t \in[0, T)$, such that $0 \in \Omega\left(t_{1}\right) \subset$ $\subset \Omega\left(t_{2}\right), 0 \leqslant t_{1}<t_{2}<T$, the function $f(z, t)=e^{t} z+\ldots$, conformally mapping $\mathbb{D}$ onto $\Omega(t)$ for each fixed $t \in[0, T)$, a.e. satisfies the (Löwner - Kufarev) equation $[9,11]$

$$
\frac{\partial f(z, t)}{\partial t}=z \frac{\partial f(z, t)}{\partial z} p(z, t), \quad z \in \mathbb{D}, \quad t \in[0, T)
$$

where, for all $t \in[0, T), p(z, t)$ is analytic in $\mathbb{D}$ with respect to $z, p(0, t)=1$, $\operatorname{Re} p(z, t)>0$ and $p(z, t)$ is measurable with respect to $t$ for any $z \in \mathbb{D}$. A similar statement can be formulated for a decreasing chain of domains.

We consider a chain of bounded domains $\Omega(t), 0 \in \Omega(t), \Omega(0)=\mathbb{D}$ with a boundary $\Gamma(t)$ and a chain $\Omega^{*}(t), \infty \in \Omega^{*}(t)$, of unbounded domains with the same boundary $\Gamma(t)$. The method of Löwner-Kufarev evolution can be used to establish a connection between conformal radii of these domains. In [6], it is shown that if $\Omega(t)$ is decreasing, $p(\cdot, t) \in C^{2}(\overline{\mathbb{D}})$ for $t \in[0, T), p(z, \cdot)$ is continuous in $[0, T)$ for $z \in \overline{\mathbb{D}}$ and $p(z, t), p^{\prime}(z, t)$ and $p^{\prime \prime}(z, t)$ are bounded in $\overline{\mathbb{D}} \times[0, T)$, then $\ln \left(r\left(\Omega^{*}(0), \infty\right)\right)=t+o(t), t \rightarrow+0$.

We suppose now, that $\Gamma(t)$ is given by the polar equation $r=\gamma(\psi, t)$. Let $G(t)$, $0 \in G(t)$ be a chain of domains bounded by a curve with the polar equation $r=\gamma^{-1}(\psi, t)$. Let $f(z, t)=a(t) z+\ldots$ and $g(z, t)=b(t) z+\ldots$ conformally map $\mathbb{D}$ onto $\Omega(t)$ and $G(t)$, respectively, where $a(t)=r(\Omega(t), 0), b(t)=r(G(t), 0)$ are positive, strictly monotone and continuous functions, $a(0)=b(0)=1$. We can always choose the parameter $t$ so that $a(t)=e^{t}\left(a(t)=e^{-t}\right.$ in the case of a decreasing chain of domains). The following theorem gives the asymptotic expansion for $b(t)$ in a neighbourhood of $t=0$.

Theorem 1. Let $\Omega(t), t \in[0, T)$, be a chain of domains (increasing or decreasing), $0 \in \Omega(t), r(\Omega(t), 0)=e^{ \pm t}$ for each $t \in[0, T), \Omega(0)=\mathbb{D}$, and the boundary $\Gamma(t)$ for each $t \in[0, T)$ given by the polar equation $r=\gamma(\psi, t), \psi \in[0,2 \pi]$, where $\gamma \in C^{3+\alpha}$, $\alpha \in(0,1)$. Let $G(t)$ be a chain of domains bounded by the family of curves with the polar equation $r=\gamma_{1}(\psi, t)=(\gamma(\psi, t))^{-1}$. Then

$$
\log r(G(t), 0)=\mp t+\left(\frac{1}{2 \pi} \int_{0}^{2 \pi}(\dot{\gamma}(\varphi, 0))^{2}-\ddot{\gamma}(\varphi, 0) d \varphi\right) t^{2}+o\left(t^{2}\right), \quad t \rightarrow+0 .
$$

By $\dot{\gamma}$, $\ddot{\gamma}$ we denote the first and second derivatives with respect to the parameter $t$, respectively. In general, we use the following convention. If $f$ is a function of a real or complex variable and $t$ is a parameter, then $\dot{f}$ denotes the derivative with respect to $t$, while $f^{\prime}$ denotes the derivative with respect to another variable.

It is not difficult to see that $r\left(\Omega^{*}(t), \infty\right)=r(G(t), 0)$ for $t \in[0, T)$. So, we have the following corollary of Theorem 1 , which is the main result of the article.

Corollary 1. Let a chain of domains $\Omega(t)$ and their boundaries $\Gamma(t)$ be the same as in Theorem 1, and $\Omega^{*}(t)$ be a chain of the unbounded components of the complement 
of $\Gamma(t)$. Then

$$
\log r\left(\Omega^{*}(t), \infty\right)=\mp t+\left(\frac{1}{2 \pi} \int_{0}^{2 \pi}(\dot{\gamma}(\varphi, 0))^{2}-\ddot{\gamma}(\varphi, 0) d \varphi\right) t^{2}+o\left(t^{2}\right), \quad t \rightarrow+0 .
$$

In Sect. 1 we deduce the integral representation for a driving function in the Löwner Kufarev equation. We use it in Sect. 2, where Theorem 1 is proved.

\section{LÖWNER-KUFAREV EQUATION}

The following theorem gives the integral representation for the driving function $p(z, t)$ in the Löwner - Kufarev equation (1). Note that we do not suppose that $f^{\prime}(0, t)=e^{ \pm t}$, as it is usually done.

Theorem 2. Let $\Omega(t), t \in[0, T)$ be a chain of domains (increasing or decreasing), $\Omega(0)=\mathbb{D}, 0 \in \Omega(t)$, with a boundary $\partial \Omega(t)$ given by the polar equation $r=\gamma(\psi, t)=1+\delta(\psi, t), \psi \in[0,2 \pi]$, where $\delta \in C^{3+\alpha}, \alpha \in(0,1)$. Let $f(z, t)=a(t) z+\ldots$ conformally map $\mathbb{D}$ onto $\Omega(t)$, a $(t)>0$. Then $f$ is differentiable with respect to $t$ for $t \in[0, T), z \in \mathbb{D}$, and satisfies the equation (1) where

$$
p(z, t)=\frac{1}{2 \pi} \int_{0}^{2 \pi} \frac{1}{\left|f^{\prime}\left(e^{i \varphi}, t\right)\right|} \dot{\delta}(\psi(\varphi, t), t) \cos (\beta(\psi(\varphi, t), t)) \frac{e^{i \varphi}+z}{e^{i \varphi}-z} d \varphi,
$$

with $\psi(\varphi, t)=\arg f\left(e^{i \varphi}, t\right)$ and $\beta(\psi, t)=-\arctan \left(\frac{\gamma^{\prime}(\psi, t)}{\gamma(\psi, t)}\right)$.

Remark 1. Note that $\beta(\psi, t)$ is an angle between a normal to the boundary $\partial \Omega(t)$ at the point $\gamma(\psi, t) e^{i \psi}$ and a radius vector of this point.

Remark 2. Here, the function $p(z, t)$ is analytic in $\mathbb{D}$ with respect to $z, \operatorname{Re} p(z, t)>0$ if $\Omega(t)$ is increasing and $\operatorname{Re} p(z, t)<0$ if $\Omega(t)$ is decreasing, $p(0, t)= \pm 1$ if $a(t)=e^{ \pm t}$.

Remark 3. Differentiating (1) with respect to $z$ and putting $z=0$, we obtain an equation for the conformal radius $r(\Omega(t), 0)=a(t)$

$$
\frac{d}{d t} \log a(t)=p(0, t)=\frac{1}{2 \pi} \int_{0}^{2 \pi} \frac{1}{\left|f^{\prime}\left(e^{i \varphi}, t\right)\right|} \dot{\delta}(\psi(\varphi, t), t) \cos (\beta(\psi(\varphi, t), t)) d \varphi .
$$

First, we prove the following lemma.

Lemma 1. Let $\Omega_{1} \subset \Omega$ be domains bounded by simple closed curves $\Gamma, \Gamma_{1}$ given by the polar equations $r=\gamma(\psi), r=\gamma_{1}(\psi), \psi \in[0,2 \pi], \gamma, \gamma_{1} \in C^{3+\alpha}, \alpha \in(0,1)$. Let $f$ and $f_{1}$ conformally map $\mathbb{D}$ onto $\Omega$ and $\Omega_{1}$, respectively, $f(0)=f_{1}(0)=0, f^{\prime}(0)>0$, $f_{1}^{\prime}(0)>0$. Let $\delta(\psi)=\gamma(\psi)-\gamma_{1}(\psi)$ satisfy $|\delta(\psi)|<\varepsilon,\left|\delta^{\prime}(\psi)\right|<\varepsilon,\left|\delta^{\prime \prime}(\psi)\right|<\varepsilon$. Then

$$
\begin{gathered}
f_{1}(z)=f\left(z\left(1-\frac{1}{2 \pi} \int_{0}^{2 \pi} \frac{1}{\left|f^{\prime}\left(e^{i \varphi}\right)\right|} \delta(\psi(\varphi)) \cos \beta(\psi(\varphi)) \frac{e^{i \varphi}+z}{e^{i \varphi}-z} d \varphi\right)\right)+ \\
+O\left(\varepsilon^{2}\right), \quad z \in \mathbb{D}, \quad \varepsilon \rightarrow+0,
\end{gathered}
$$

with $\psi(\varphi)=\arg f\left(e^{i \varphi}\right)$ and $\beta(\psi)=-\arctan \left(\frac{\gamma^{\prime}(\psi)}{\gamma(\psi)}\right)$. 
We need the following theorem obtained by Siryk [12] (see also [13, p. 371]). It provides the asymptotic representation for functions conformally mapping $\mathbb{D}$ onto domains close to $\mathbb{D}$.

Theorem 3. [12] Let $\Omega$ be a domain that contains 0 and is bounded by a curve given by the polar equation $r=1-\delta(\psi), 0 \leqslant \psi \leqslant 2 \pi$, where $\psi$ is twice differentiable and satisfies the conditions

$$
|\delta(\psi)|<\varepsilon, \quad\left|\delta^{\prime}(\psi)\right|<\varepsilon, \quad\left|\delta^{\prime \prime}(\psi)\right|<\varepsilon
$$

Then a function $f: \mathbb{D} \rightarrow \Omega, f(0)=0, f^{\prime}(0)>0$, mapping $\mathbb{D}$ conformally onto $\Omega$ has the asymptotic representation

$$
f(z)=z\left(1-\frac{1}{2 \pi} \int_{0}^{2 \pi} \delta(\psi) \frac{e^{i \psi}+z}{e^{i \psi}-z} d \psi\right)+O\left(\varepsilon^{2}\right), \quad \varepsilon \rightarrow+0 .
$$

Proof of Lemma 1. We denote the inverse function by $g=f^{-1}$. Since $\gamma \in C^{3+\alpha}$, $f, f^{\prime}, f^{\prime \prime}, f^{(3)}$ can be continuously extended to $\overline{\mathbb{D}}\left[14\right.$, p. 49] and $f^{\prime}$ does not vanish there $\left[14\right.$, p. 48]. Hence $g, g^{\prime}, g^{\prime \prime}, g^{(3)}$ can be continuously extended to $\overline{\mathbb{D}}$.

The function $g$ maps the curve $\Gamma_{1}$ onto a simple closed curve in $\mathbb{D}$, which has the following equation

$$
\omega(\varphi)=g\left(\gamma_{1}(\psi(\varphi)) e^{i \psi(\varphi)}\right)=g\left(f\left(e^{i \varphi}\right)-\delta(\psi(\varphi)) e^{i \psi(\varphi)}\right), \quad 0 \leqslant \varphi \leqslant 2 \pi .
$$

We have

$$
\begin{gathered}
\omega(\varphi)-e^{i \varphi}=g\left(f\left(e^{i \varphi}\right)-\delta(\psi(\varphi)) e^{i \psi(\varphi)}\right)-g\left(f\left(e^{i \varphi}\right)\right)= \\
=-g^{\prime}\left(f\left(e^{i \varphi}\right)\right) \delta(\psi(\varphi)) e^{i \psi(\varphi)}+O\left(\varepsilon^{2}\right)=-\frac{1}{f^{\prime}\left(e^{i \varphi}\right)} \delta(\psi(\varphi)) e^{i \psi(\varphi)}+O\left(\varepsilon^{2}\right)= \\
=-\frac{1}{\left|f^{\prime}\left(e^{i \varphi}\right)\right|} \delta(\psi(\varphi)) e^{i(\varphi-\beta(\psi(\varphi)))}+O\left(\varepsilon^{2}\right), \quad \varepsilon \rightarrow+0,
\end{gathered}
$$

where $\beta(\psi(\varphi))=\arg \frac{f^{\prime}\left(e^{i \varphi}\right) e^{i \varphi}}{f\left(e^{i \varphi}\right)}=-\arctan \frac{\gamma^{\prime}(\psi(\varphi))}{\gamma(\psi(\varphi))}$. Differentiating (7), we obtain

$$
\begin{gathered}
\omega^{\prime}(\varphi)=g^{\prime}\left(f\left(e^{i \varphi}\right)-\delta(\psi(\varphi)) e^{i \psi(\varphi)}\right)\left[f^{\prime}\left(e^{i \varphi}\right) i e^{i \varphi}-\psi^{\prime}(\varphi) e^{i \psi(\varphi)}\left(\delta^{\prime}(\psi(\varphi))+i \delta(\psi(\varphi))\right)\right], \\
\omega^{\prime \prime}(\varphi)=g^{\prime \prime}\left(f\left(e^{i \varphi}\right)-\delta(\psi(\varphi)) e^{i \psi(\varphi)}\right)\left[f^{\prime}\left(e^{i \varphi}\right) i e^{i \varphi}-\psi^{\prime}(\varphi) e^{i \psi(\varphi)}\left(\delta^{\prime}(\psi(\varphi))+i \delta(\psi(\varphi))\right)\right]^{2}+ \\
+g^{\prime}\left(f\left(e^{i \varphi}\right)-\delta(\psi(\varphi)) e^{i \psi(\varphi)}\right)\left[-f^{\prime \prime}\left(e^{i \varphi}\right) e^{2 i \varphi}-f^{\prime}\left(e^{i \varphi}\right) e^{i \varphi}-\right. \\
\left.-\psi^{\prime \prime}(\varphi) e^{i \psi(\varphi)}\left(\delta^{\prime}(\psi(\varphi))+i \delta(\psi(\varphi))\right)-\left(\psi^{\prime}(\varphi)\right)^{2} e^{i \psi(\varphi)}\left(\delta^{\prime \prime}(\psi(\varphi))+2 i \delta^{\prime}(\psi(\varphi))-\delta(\psi(\varphi))\right)\right] .
\end{gathered}
$$

Since, $g^{\prime \prime}, g^{(3)}$ can be continuously extended to $\overline{\mathbb{D}}$ and $|\delta(\psi)|<\varepsilon,\left|\delta^{\prime}(\psi)\right|<\varepsilon$, $\left|\delta^{\prime \prime}(\psi)\right|<\varepsilon$ we obtain the following estimates

$$
\begin{gathered}
\omega^{\prime}(\varphi)=\left[g^{\prime}\left(f\left(e^{i \varphi}\right)\right)+O(\varepsilon)\right]\left[f^{\prime}\left(e^{i \varphi}\right) i e^{i \varphi}+O(\varepsilon)\right]=i e^{i \varphi}+O(\varepsilon), \quad \varepsilon \rightarrow+0, \\
\omega^{\prime \prime}(\varphi)=\left[g^{\prime \prime}\left(f\left(e^{i \varphi}\right)\right)+O(\varepsilon)\right]\left[f^{\prime}\left(e^{i \varphi}\right) i e^{i \varphi}+O(\varepsilon)\right]^{2}+\left[g^{\prime}\left(f\left(e^{i \varphi}\right)\right)+O(\varepsilon)\right]\left[-f^{\prime \prime}\left(e^{i \varphi}\right) e^{2 i \varphi}-\right.
\end{gathered}
$$




$$
\begin{gathered}
\left.-f^{\prime}\left(e^{i \varphi}\right) e^{i \varphi}+O(\varepsilon)\right]=\left[-\frac{f^{\prime \prime}\left(e^{i \varphi}\right)}{\left(f^{\prime}\left(e^{i \varphi}\right)\right)^{3}}+O(\varepsilon)\right]\left[-\left(f^{\prime}\left(e^{i \varphi}\right)\right)^{2} e^{2 i \varphi}+O(\varepsilon)\right]+ \\
+\left[\frac{1}{f^{\prime}\left(e^{i \varphi}\right)}+O(\varepsilon)\right]\left[-f^{\prime \prime}\left(e^{i \varphi}\right) e^{2 i \varphi}-f^{\prime}\left(e^{i \varphi}\right) e^{i \varphi}+O(\varepsilon)\right]= \\
=\frac{f^{\prime \prime}\left(e^{i \varphi}\right)}{f^{\prime}\left(e^{i \varphi}\right)} e^{2 i \varphi}-\frac{f^{\prime \prime}\left(e^{i \varphi}\right)}{f^{\prime}\left(e^{i \varphi}\right)} e^{2 i \varphi}-e^{i \varphi}+O(\varepsilon)=-e^{i \varphi}+O(\varepsilon), \quad \varepsilon \rightarrow+0 .
\end{gathered}
$$

Dividing (9) by $\omega(\varphi)$ gives

$$
\frac{\partial}{\partial \varphi} \log \omega(\varphi)=\frac{i e^{i \varphi}+O(\varepsilon)}{e^{i \varphi}+O(\varepsilon)}=i+O(\varepsilon), \quad \varepsilon \rightarrow+0
$$

therefore

$$
\frac{\partial}{\partial \varphi} \arg \omega(\varphi)=1+O(\varepsilon), \quad \varepsilon \rightarrow+0
$$

Hence, the curve $g\left(\Gamma_{1}\right)$ can be given by a polar equation for $\varepsilon$ small enough. Denote $\mu(\varphi)=\arg \omega(\varphi)-\varphi, 0 \leqslant \varphi \leqslant 2 \pi$. Using (8) we obtain

$$
\begin{gathered}
\mu(\varphi)=\arg \frac{g\left(f\left(e^{i \varphi}\right)-\delta(\psi(\varphi)) e^{i \psi(\varphi)}\right)}{e^{i \varphi}}=\arg \left(1+\frac{g\left(f\left(e^{i \varphi}\right)-\delta(\psi(\varphi)) e^{i \psi(\varphi)}\right)-e^{i \varphi}}{e^{i \varphi}}\right)= \\
=\arg \left(1-\frac{1}{\left|f^{\prime}\left(e^{i \varphi}\right)\right|} \delta(\psi(\varphi)) e^{-i \beta(\psi(\varphi))}+O\left(\varepsilon^{2}\right)\right), \quad \varepsilon \rightarrow+0,
\end{gathered}
$$

Therefore, it is not difficult to see that $\mu(\varphi)=O\left(\varepsilon^{2}\right)$. From (12) we conclude that $\mu^{\prime}(\varphi)=O(\varepsilon)$. Let $r=1-\Delta(\varphi)$ be the polar equation of $g\left(\Gamma_{1}\right)$. Then $\Delta(\varphi)=1-\left|\omega\left(\varphi_{1}\right)\right|$, where $\varphi_{1}$ is a unique solution of $\arg \omega\left(\varphi_{1}\right)=\varphi$. Hence $\mu\left(\varphi_{1}\right)+\varphi_{1}=\varphi$ and $\varphi_{1}=\varphi+O\left(\varepsilon^{2}\right)$. Applying (8) gives

$$
\begin{gathered}
\Delta(\varphi)=1-\left|\omega\left(\varphi_{1}\right)\right|=1-\left|\omega\left(\varphi+O\left(\varepsilon^{2}\right)\right)\right|=1-\left|\omega(\varphi)+O\left(\varepsilon^{2}\right)\right|= \\
=1-\left|e^{i \varphi}+\left(\omega(\varphi)-e^{i \varphi}\right)+O\left(\varepsilon^{2}\right)\right|=1-\left|e^{i \varphi}-\frac{1}{\left|f^{\prime}\left(e^{i \varphi}\right)\right|} \delta(\psi(\varphi)) e^{i(\varphi-\beta(\psi(\varphi)))}+O\left(\varepsilon^{2}\right)\right|= \\
=1-\left|1-\frac{\delta(\psi(\varphi))}{\left|f^{\prime}\left(e^{i \varphi}\right)\right|} e^{-i \beta(\psi(\varphi)))}+O\left(\varepsilon^{2}\right)\right|= \\
=\frac{\delta(\psi(\varphi))}{\left|f^{\prime}\left(e^{i \varphi}\right)\right|} \cos \beta(\psi(\varphi))+O\left(\varepsilon^{2}\right), \quad 0 \leqslant \varphi \leqslant 2 \pi, \quad \varepsilon \rightarrow+0 .
\end{gathered}
$$

From (13) it easily follows that $|\Delta(\varphi)|=O(\varepsilon)$. Now we want to deduce similar estimates for $\left|\Delta^{\prime}(\varphi)\right|,\left|\Delta^{\prime \prime}(\varphi)\right|$. It follows from (8)-(10) that $|\omega(\varphi)|^{\prime}=O(\varepsilon),|\omega(\varphi)|^{\prime \prime}=O(\varepsilon)$. Differentiating the equation $\Delta(\varphi+\mu(\varphi))=1-|\omega(\varphi)|$ we obtain

$$
\begin{gathered}
\Delta^{\prime}(\varphi+\mu(\varphi))\left(1+\mu^{\prime}(\varphi)\right)=-|\omega(\varphi)|^{\prime}, \\
\Delta^{\prime \prime}(\varphi+\mu(\varphi))\left(1+\mu^{\prime}(\varphi)\right)^{2}+\Delta^{\prime}(\varphi+\mu(\varphi)) \mu^{\prime \prime}(\varphi)=-|\omega(\varphi)|^{\prime \prime}
\end{gathered}
$$

Hence $\left|\Delta^{\prime}(\varphi)\right|=O(\varepsilon),\left|\Delta^{\prime \prime}(\varphi)\right|=O(\varepsilon)$.

Thus, the curve $g\left(\Gamma_{1}\right)$ has the polar equation $r=1-\Delta(\varphi)$ where $|\Delta(\varphi)|=O(\varepsilon)$, $\left|\Delta^{\prime}(\varphi)\right|=O(\varepsilon),\left|\Delta^{\prime \prime}(\varphi)\right|=O(\varepsilon), \varepsilon \rightarrow+0$. Therefore, we can apply Theorem 3 for 
$h=g \circ f_{1}, h(0)=0, h^{\prime}(0)>0$, conformally mapping $\mathbb{D}$ onto the domain bounded by $g\left(\Gamma_{1}\right)$. Hence, (6) gives

$$
h(z)=z\left(1-\frac{1}{2 \pi} \int_{0}^{2 \pi} \frac{\delta(\psi(\varphi))}{\left|f^{\prime}\left(e^{i \varphi}\right)\right|} \cos \beta(\psi(\varphi)) \frac{e^{i \varphi}+z}{e^{i \varphi}-z} d \varphi\right)+O\left(\varepsilon^{2}\right), \quad z \in \mathbb{D}, \quad \varepsilon \rightarrow+0 .
$$

Since $f_{1}=f \circ h$, we obtain (5).

Proof of Theorem 2. Let $\Omega(t)$ be an increasing chain. Fix $t \in[0, T), h$ such that $t+h \in[0, T)$. First, let $h$ be negative, so $\Omega(t+h) \in \Omega(t)$. Denote $\lambda(\psi, h)=$ $=\gamma(\psi, t)-\gamma(\psi, t+h)$. Since $\gamma \in C^{3+\alpha}$, we conclude that $|\lambda(\psi, h)|=O(h)$, $\left|\lambda^{\prime}(\psi, h)\right|=O(h),\left|\lambda^{\prime \prime}(\psi, h)\right|=O(h)$. So we can apply Lemma 1. By (5), we obtain

$$
\begin{gathered}
f(z, t+h)=f\left(z\left(1-\int_{0}^{2 \pi} s(\varphi, z, t) \lambda(\psi(\varphi, t), h) d \varphi\right), t\right)+o(h)= \\
\quad=f\left(z\left(1+h \int_{0}^{2 \pi} s(\varphi, z, t) \dot{\delta}(\psi(\varphi, t), t) d \varphi\right), t\right)+o(h),
\end{gathered}
$$

where

$$
s(\varphi, z, t)=\frac{1}{2 \pi} \frac{1}{\left|f^{\prime}\left(e^{i \varphi}, t\right)\right|} \cos (\beta(\psi(\varphi, t), t)) \frac{e^{i \varphi}+z}{e^{i \varphi}-z} .
$$

Therefore,

$$
\begin{gathered}
f(z, t+h)-f(z, t)=f\left(z+z h \int_{0}^{2 \pi} s(\varphi, z, t) \dot{\delta}(\psi(\varphi, t), t) d \varphi, t\right)-f(z, t)+o(h)= \\
=f^{\prime}(z, t) z h \int_{0}^{2 \pi} s(\varphi, z, t) \dot{\delta}(\psi(\varphi, t), t) d \varphi+o(h), \quad h \rightarrow 0 .
\end{gathered}
$$

Let now $h$ be positive. Then $\Omega(t) \in \Omega(t+h)$, and $\lambda(\psi, h)=\gamma(\psi, t+h)-\gamma(\psi, t)$ satisfies all the conditions of Lemma 1 . So we have

$$
\begin{gathered}
f(z, t)=f\left(z\left(1-\int_{0}^{2 \pi} s(\varphi, z, t+h) \lambda(\psi(\varphi, t+h), h) d \varphi\right), t+h\right)+o(h)= \\
=f\left(z\left(1-h \int_{0}^{2 \pi} s(\varphi, z, t+h) \dot{\delta}(\psi(\varphi, t+h), t) d \varphi\right), t+h\right)+o(h),
\end{gathered}
$$

So we obtain

$$
\begin{gathered}
f(z, t+h)-f(z, t)=f(z, t+h)- \\
-f\left(z-z h \int_{0}^{2 \pi} s(\varphi, z, t+h) \dot{\delta}(\psi(\varphi, t+h), t) d \varphi, t+h\right)+o(h)=
\end{gathered}
$$




$$
=f^{\prime}(z, t+h) z h \int_{0}^{2 \pi} s(\varphi, z, t+h) \dot{\delta}(\psi(\varphi, t+h), t) d \varphi+o(h), \quad h \rightarrow 0,
$$

Thus, we have shown that $f$ is differentiable with respect to $t$ and satisfies (1). One can similarly repeat the proof for a decreasing chain of domains.

\section{PROOF OF THEOREM 1}

Let, for each $t \in[0, T), f(\cdot, t)$ and $g(\cdot, t)$ conformally map $\mathbb{D}$ onto $\Omega(t)$ and $G(t)$, respectively, $f(0, t)=g(0, t)=0, f^{\prime}(0, t)>0, g^{\prime}(0, t)>0$. Denote $\delta(\psi, t)=\gamma(\psi, t)-1$, $\delta_{1}(\psi, t)=\gamma_{1}(\psi, t)-1$. By Theorem 2 and Remark 3, conformal radii satisfy the equations

$$
\frac{d}{d t} \log r(\Omega(t), 0)=p(0, t), \quad \frac{d}{d t} \log r(G(t), 0)=q(0, t),
$$

where $p(z, t), q(z, t)$ are given by

$$
\begin{gathered}
p(z, t)=\frac{1}{2 \pi} \int_{0}^{2 \pi} \frac{1}{\left|f^{\prime}\left(e^{i \varphi}, t\right)\right|} \dot{\delta}(\psi(\varphi, t), t) \cos (\beta(\psi(\varphi, t), t)) \frac{e^{i \varphi}+z}{e^{i \varphi}-z} d \varphi, \\
q(z, t)=\frac{1}{2 \pi} \int_{0}^{2 \pi} \frac{1}{\left|g^{\prime}\left(e^{i \varphi}, t\right)\right|} \dot{\delta}_{1}\left(\psi_{1}(\varphi, t), t\right) \cos \left(\beta_{1}\left(\psi_{1}(\varphi, t), t\right)\right) \frac{e^{i \varphi}+z}{e^{i \varphi}-z} d \varphi,
\end{gathered}
$$

with $\psi(\varphi, t)=\arg f\left(e^{i \varphi}, t\right), \quad \beta(\psi, t)=-\arctan \left(\frac{\gamma^{\prime}(\psi, t)}{\gamma(\psi, t)}\right), \quad \psi_{1}(\varphi, t)=\arg g\left(e^{i \varphi}, t\right)$, $\beta_{1}(\psi, t)=-\arctan \left(\frac{\gamma_{1}^{\prime}(\psi, t)}{\gamma_{1}(\psi, t)}\right)$. First, we want to prove the following equalities

$$
\begin{gathered}
p(z, 0)=-q(z, 0)=\frac{1}{2 \pi} \int_{0}^{2 \pi} \dot{\delta}(\varphi, 0) \frac{e^{i \varphi}+z}{e^{i \varphi}-z} d \varphi, \quad z \in \mathbb{D}, \\
\frac{\partial q(z, 0)}{\partial t}-\frac{\partial p(z, 0)}{\partial t}=\frac{1}{\pi} \int_{0}^{2 \pi}(\dot{\delta}(\varphi, 0))^{2}-\ddot{\delta}(\varphi, 0) \frac{e^{i \varphi}+z}{e^{i \varphi}-z} d \varphi, \quad z \in \mathbb{D} .
\end{gathered}
$$

Elementary calculations lead us to the formulas

$$
\begin{gathered}
\dot{\delta}_{1}(\psi, 0)=-\dot{\delta}(\psi, 0), \\
\dot{\delta}_{1}^{\prime}(\psi, 0)=-\dot{\delta}^{\prime}(\psi, 0), \\
\ddot{\delta}_{1}(\psi, 0)=-\ddot{\delta}(\psi, 0)+2(\dot{\delta}(\psi, 0))^{2} .
\end{gathered}
$$

Since $f^{\prime}\left(e^{i \varphi}, 0\right)=1, \psi(\varphi, 0)=\varphi, \beta(\psi, 0)=0$, representation (15) gives

$$
p(z, 0)=\frac{1}{2 \pi} \int_{0}^{2 \pi} \dot{\delta}(\varphi, 0) \frac{e^{i \varphi}+z}{e^{i \varphi}-z} d \varphi, \quad z \in \mathbb{D} .
$$

Similarly we obtain

$$
q(z, 0)=\frac{1}{2 \pi} \int_{0}^{2 \pi} \dot{\delta}_{1}(\varphi, 0) \frac{e^{i \varphi}+z}{e^{i \varphi}-z} d \varphi, \quad z \in \mathbb{D} .
$$

Thus, (19) gives (17). 
Denote by $P(t)$ the expression under the integral in (15). Elementary calculations yield the following result

$$
\dot{P}(0)=-\left.\frac{\partial}{\partial t}\left(\left|f^{\prime}\left(e^{i \varphi}, t\right)\right|\right)\right|_{t=0} \dot{\delta}(\varphi, 0)+\dot{\delta}^{\prime}(\varphi, 0) \dot{\psi}(\varphi, 0)+\ddot{\delta}(\varphi, 0) .
$$

Similarly, we denote by $Q(t)$ the expression under the integral in (16) and obtain

$$
\dot{Q}(0)=-\left.\frac{\partial}{\partial t}\left(\left|g^{\prime}\left(e^{i \varphi}, t\right)\right|\right)\right|_{t=0} \dot{\delta}_{1}(\varphi, 0)+\dot{\delta}_{1}^{\prime}(\varphi, 0) \dot{\psi}_{1}(\varphi, 0)+\ddot{\delta}_{1}(\varphi, 0) .
$$

It easily follows from (1) that $\dot{\psi}(\varphi, 0)=\operatorname{Im} p\left(e^{i \varphi}, 0\right), \dot{\psi}_{1}(\varphi, 0)=\operatorname{Im} q\left(e^{i \varphi}, 0\right)$. Therefore, (17) gives

$$
\dot{\psi}_{1}(\varphi, 0)=-\dot{\psi}(\varphi, 0), \quad 0 \leqslant \varphi \leqslant 2 \pi .
$$

Differentiating (1) with respect to $z$ and putting $t=0$ we conclude that $\left.\frac{\partial^{2} f(z, t)}{\partial z \partial t}\right|_{t=0}=$ $=p(z, 0)+z p^{\prime}(z, 0)$. Hence

$$
\dot{f}^{\prime}\left(e^{i \varphi}, 0\right)=\left.\frac{\partial}{\partial t} f^{\prime}\left(e^{i \varphi}, t\right)\right|_{t=0}=p\left(e^{i \varphi}, 0\right)+e^{i \varphi} p^{\prime}\left(e^{i \varphi}, 0\right) .
$$

Since

$$
f^{\prime}\left(e^{i \varphi}, t\right)=f^{\prime}\left(e^{i \varphi}, 0\right)+\dot{f}^{\prime}\left(e^{i \varphi}, 0\right) t+o(t), \quad t \rightarrow+0,
$$

we see that

$$
\begin{gathered}
\left|f^{\prime}\left(e^{i \varphi}, t\right)\right|=\left|e^{i \varphi}+\dot{f}^{\prime}\left(e^{i \varphi}, 0\right) t\right|+o(t)= \\
=1+\left|\dot{f}^{\prime}\left(e^{i \varphi}, 0\right)\right| \cos \left(\arg \left(\dot{f}^{\prime}\left(e^{i \varphi}, 0\right)\right)-\varphi\right) t+o(t), \quad t \rightarrow+0 .
\end{gathered}
$$

Thus,

$$
\left.\frac{\partial}{\partial t}\left|f^{\prime}\left(e^{i \varphi}, t\right)\right|\right|_{t=0}=\left|\dot{f}^{\prime}\left(e^{i \varphi}, 0\right)\right| \cos \left(\arg \left(\dot{f}^{\prime}\left(e^{i \varphi}, 0\right)\right)-\varphi\right), \quad 0 \leqslant \varphi \leqslant 2 \pi .
$$

From (25) and (17) we deduce

$$
\left.\frac{\partial}{\partial t}\left|g^{\prime}\left(e^{i \varphi}, t\right)\right|\right|_{t=0}=-\left.\frac{\partial}{\partial t}\left|f^{\prime}\left(e^{i \varphi}, t\right)\right|\right|_{t=0}, \quad 0 \leqslant \varphi \leqslant 2 \pi .
$$

Formulas (19)-(24) and (26) show that $\dot{Q}(0)-\dot{P}(0)=-2 \ddot{\delta}(\psi, 0)+2(\dot{\delta}(\psi, 0))^{2}$, which leads to (18). One can deduce (2) from (14) and (17), (18). Indeed, let, for example, $\Omega(t)$ be an increasing chain of domains. Using (14) and (17) we obtain

$$
\left.\frac{d}{d t} \log r(G(t), 0)\right|_{t=0}=q(0,0)=-p(0,0)=-1,
$$

Similarly, using (18) we obtain

$$
\left.\frac{d^{2}}{d t^{2}} \log r(G(t), 0)\right|_{t=0}=\left.\frac{d}{d t} q(0, t)\right|_{t=0}=\frac{1}{\pi} \int_{0}^{2 \pi}(\dot{\delta}(\varphi, 0))^{2}-\ddot{\delta}(\varphi, 0) d \varphi .
$$

Acknowledgements: This work was supported by the Russian Science Foundation (project no. 17-11-01229). 


\title{
References
}

1. Lebedev N. A. Printsip ploshchadei v teorii odnolistnykh funktsii [The area principle in the theory of univalent functions]. Moscow, Nauka, 1975. 336 p. (in Russian).

2. Hamilton D. H. Conformal welding. Handbook of complex analysis: geometric function theory. Ed. R. Kühnau. Amsterdam, North Holland, 2002. 548 p.

3. Grong E., Gumenyuk P., Vasil'ev A. Matching univalent functions and conformal welding. Ann. Acad. Sci. Fenn. Math., 2009, vol. 34, pp. 303-314.

4. Bishop C. J. Conformal welding and Koebe's theorem. Ann. Math., 2007, vol. 166, pp. 613656. DOI: https://doi.org/10.4007/annals.2007.166.613

5. Prokhorov D. V. Conformal welding for domains close to a disk. Anal. Math. Phys., 2011, vol. 1, pp. 101-114. DOI: https://doi.org/10.1007/s13324-011-0007-0

6. Prokhorov D. V. Asymptotic conformal welding via Löwner-Kufarev evolution. Comput. Methods Funct. Theory, 2013, vol. 13, no. 1, pp. 37-46. DOI: https://doi.org/10.1007/s40315-012-0002-y

7. Marshall D. E. Conformal Welding for Finitely Connected Regions. Comput. Methods Funct. Theory, 2012, vol. 11, pp. 655-669. DOI: https://doi.org/10.1007/BF03321879

8. Löwner K. Untersuchungen über schlichte konforme Abbildungen des Einheitskreises. I. Math. Ann., 1923, vol. 89, pp. 103-121.

9. Kufarev P. P. Ob odnoparametricheskikh semeistvakh analiticheskikh funktsii [On oneparametric families of analytic functions]. Mat. Sbornik, 1943, vol. 13(55), no. 1, pp. 87118 (in Russian).

10. Pommerenke Ch. Über die Subordination analytischer Funktionen. J. Reine Angew. Math., 1965, vol. 218, pp. 159-173. DOI: https://doi.org/10.1515/crll.1965.218.159

11. Pommerenke Ch. Univalent functions. Gottingen, Vandenhoeck and Ruprecht, 1975. 376 p.

12. Siryk G. V. O konformnom otobrazhenii blizkikh oblastei [On a conformal mapping of near domains]. Uspekhi Mat. Nauk, 1956, vol. 11, no. 5(71), pp. 57-60 (in Russian).

13. Lavrentyev M. A., Shabat B. V. Metody teorii funktsii kompleksnogo peremennogo [Methods of Function Theory of a Complex Variable]. Moscow, Nauka, 1965. 716 p. (in Russian).

14. Pommerenke Ch. Boundary Behaviour of Conformal Maps. Berlin, Springer-Verlag, 1992. 300 p. DOI: https://doi.org/10.1007/978-3-662-02770-7

\section{Cite this article as:}

Zherdev A. V. An Asymptotic Relation for Conformal Radii of Two Nonoverlapping Domains. Izv. Saratov Univ. (N. S.), Ser. Math. Mech. Inform., 2018, vol. 18, iss. 3, pp. 274-283. DOI: https://doi.org/10.18500/1816-9791-2018-18-3-274-283

УДК 517.54

\section{АСИМПТОТИЧЕСКОЕ СООТНОШЕНИЕ ДЛЯ КОНФОРМНЫХ РАДИУСОВ ДВУХ НЕНАЛЕГАЮЩИХ ОБЛАСТЕЙ}

\begin{abstract}
А. В. Жердев
Жердев Андрей Владимирович, аспирант касредры математического анализа, Саратовский национальный исследовательский государственный университет имени Н. Г. Чернышевского, Россия, 410012, Саратов, Астраханская, 83; Петрозаводский государственный университет, Россия, 185910, Республика Карелия, Петрозаводск, просп. Ленина, 33, jerdevandrey@gmail.com
\end{abstract}


В статье рассматривается семейство замкнутых жордановых кривых, заданных в полярной системе координат и непрерывно зависящих от параметра, и такое, что области, ограниченные этими кривыми, образуют возрастающее или убывающее семейство. Такое семейство областей описывается дифрференциальным уравнением Левнера - Кусрарева. Для рассмотренного случая получено интегральное представление для управляющей фрункции в этом уравнении. Используя это представление, получено асимптотическое соотношение, связывающее консрормные радиусы ограниченной и неограниченной компоненты дополнения к жордановой кривой, когда ограниченная компонента близка к единичному кругу.

Ключевые слова: уравнение Левнера - Куфарева, консрормный радиус, асимптотическое разложение, неналегающие области.

Благодарности. Работа выполнена при финансовой поддержке Российского научного фонда (проект № 17-11-01229).

\section{Образец для цитирования:}

Zherdev A. V. An Asymptotic Relation for Conformal Radii of Two Nonoverlapping Domains [Жердев А. В. Асимптотическое соотношение для конформных радиусов двух неналегающих областей] // Изв. Сарат. ун-та. Нов. сер. Сер. Математика. Механика. Информатика. 2018. T. 18, вып. 3. С. 274-283. DOI: https://doi.org/10.18500/1816-9791-2018-18-3-274-283 